\title{
EXTRAÇÃO DO ÓLEO DA AMÊNDOA DA MACAÚBA (ACROCOMIA ACULEATA) UTILIZANDO DIÓXIDO DE CARBONO SUPERCRÍTICO
}

\author{
C. P. TRENTINI ${ }^{1}$, K. A. SANTOS ${ }^{2}$, L. CARDOZO-FILHO ${ }^{3}$, E. A. SILVA $^{2}$ e C. SILVA ${ }^{1}$ \\ ${ }^{1}$ Universidade Estadual de Maringá, Departamento de Tecnologia \\ ${ }^{2}$ Universidade Estadual do Oeste do Paraná, Departamento de Tecnologia \\ ${ }^{3}$ Universidade Estadual de Maringá, Departamento de Engenharia Química \\ E-mail para contato: camiladasilva.eq@ gmail.com
}

\begin{abstract}
RESUMO - O presente trabalho teve como objetivo avaliar a extração do óleo da amêndoa da macaúba utilizando $\mathrm{CO}_{2}$ supercrítico como solvente. Nos experimentos adotou-se um planejamento experimental $2^{3}$ para avaliar o efeito da temperatura $\left(40\right.$ a $\left.80{ }^{\circ} \mathrm{C}\right)$ e pressão (180 a 220 bar) no rendimento da extração, com vazão constante de solvente em $3 \mathrm{~mL} \mathrm{~min}^{-1} \mathrm{e}$ tempo total de extração de 200 minutos. A modelagem matemática da cinética de extração foi realizada empregando o modelo de Sovová, o qual se ajustou adequadamente aos dados experimentais. As variáveis temperatura e pressão apresentaram efeito significativo $(\mathrm{p}>0,05)$ no rendimento. Com o aumento da pressão ocorre o aumento do rendimento devido aos maiores valores densidade do $\mathrm{CO}_{2}$, sendo que o aumento da temperatura reduz a densidade do solvente e ocasiona redução no rendimento da extração. A condição experimental de $40{ }^{\circ} \mathrm{C}$ e 220 bar forneceu rendimentos de $\sim 42 \%$ em óleo, sendo que esta condição apresentou $>93 \%$ do rendimento obtido pelo método convencional com $n$ hexano e diclorometeno como solvente e corresponde a condição que apresentou maior solubilidade do óleo no $\mathrm{CO}_{2}$.
\end{abstract}

\section{INTRODUÇÃO}

A palmeira da macaúba é uma importante fonte de recursos, os quais são utilizados como combustíveis, medicamentos caseiros, na cobertura das casas ou confecção de utensílios e adornos domésticos, e em alguns casos como matéria-prima para as indústrias locais (Lorenzi e Negrelle, 2006; Ciconini et al., 2013). Entretanto, o produto economicamente mais expressivo desta palmeira é o fruto, principalmente devido aos óleos que produz que representa cerca de $30 \%$ de cada fruto. Seu potencial oleaginoso se compara ou supera o potencial das plantas mais produtivas (Lorenzi e Negrelle, 2006; Ciconini et al., 2013).

$\mathrm{Na}$ extração de óleos vegetais por solventes orgânicos, normalmente, se utiliza o Soxhlet, sendo que o solvente comumente utilizado é o $n$-hexano, o qual figura como um recurso não renovável, com elevada inflamabilidade e toxicidade (Merck, 2006). Este método ocasiona a produção de resíduos indesejáveis, requer a remoção do solvente no final do processo de extração, gerando impacto ambiental, alto custo e tempo de processamento. Além do que, a alta temperatura para remoção do solvente remanescente no extrato, pode afetar a qualidade organoléptica do material (Oetterer, et al., 2006). Nos últimos anos tem se enfatizado ligação entre a gestão da tecnologia e a do meio ambiente nas empresas, com o 
crescimento da aplicação da química verde (Prado, 2003; Sun et al., 2012). Desta forma, a busca por solventes e métodos alternativos para extração de óleos vegetais e compostos bioativos presentes em matrizes oleaginosas é foco de interesse.

A extração por fluido supercrítico é descrita na literatura como uma técnica alternativa aos métodos clássicos de extração (Arias et al., 2009; Corso et al.; 2010; Pederssetti et al., 2010; Nimet et al., 2011). No estado supercrítico, o fluido adquire uma densidade similar à de um líquido (portanto um poder de solvatação) e simultaneamente uma compressibilidade parecida com a de um gás. O dióxido de carbono e o propano são os solventes mais utilizados para a extração a partir de fluido supercrítico. A extração supercrítica utilizando dióxido de carbono e propano é menos sujeito à oxidação do que o óleo extraído utilizando o método convencional, em que $n$-hexano é utilizado como solvente na extração (Nimet et al., 2011).

O dióxido de carbono é comumente usado para a extração a partir de fluido supercrítico, principalmente devido às suas propriedades físicas e químicas, tais como pressão ( 73,82 bar) e temperatura crítica baixa $\left(31^{\circ} \mathrm{C}\right)$ (Reverchon e de Marco, 2006). Além disso, apresenta vantagens em relação aos solventes orgânicos, pois apresenta características de gás e líquido, e não tem a necessidade de etapas posteriores para a remoção do solvente (Corso et al., 2010; Huisden et al., 2010).

Com base no contexto descrito, o presente trabalho teve como objetivo avaliar a extração do óleo da amêndoa da macaúba utilizando $\mathrm{CO}_{2}$ supercrítico como solvente, avaliando o efeito da temperatura e pressão no rendimento em óleo, bem como a modelagem dos dados experimentais empregando o modelo de Sovová.

\section{MATERIAIS E MÉTODOS}

\subsection{Materiais}

Foram utilizados frutos de Macaúba da variedade: Acrocomia aculeata, coletados na região do Cariri no estado do Ceará, Brasil. Para a extração do óleo utilizou-se dióxido de carbono (99\%) de pureza com procedente White Martins; para a extração convencional (Soxhlet) foi utilizado $n$-hexano e diclorometano $(99,5 \%)$ de procedência F. Maia.

\subsection{Procedimentos Experimentais}

Preparo da matéria-prima: Os frutos da macaúba foram despolpados com o auxílio de faca inox sobre uma bandeja separando a casca, polpa, amêndoa e endocarpo. O material foi pesado, acondicionado em embalagens de plástico e armazenados em freezer a $-20^{\circ} \mathrm{C}$. As amostras foram mantidas congeladas até o momento da secagem. As amostras foram secas em estufa com circulação de ar a $60{ }^{\circ} \mathrm{C}$ por 4 horas. A amostra seca foi triturada em um moinho elétrico (IKA, modelo A 11/B) na velocidade de 14000 RPM e utilizou-se peneiras do tipo Tyler para a classificação granulométrica das amostras, com auxílio de um agitador mecânico de peneiras (Bertel, série 1.0), obtendo amostras com diâmetro médio de 0,841 $\mathrm{mm}$.

Extração por Soxhlet: A extração foi realizada conforme recomendado por Instituto Adolfo Lutz (2004). Foi utilizado tempo de extração de 480 minutos e 5 gramas de material. A temperatura de extração do Soxhlet foi mantida constante acima da temperatura de refluxo do solvente em todos os ensaios. Os ensaios foram realizados em duplicata. Após o tempo 
de extração o solvente remanescente no balão foi evaporado em estufa com circulação a 60 ${ }^{\circ} \mathrm{C}$ até peso constante.

Extração por Fluido Supercrítico: A extração em condições pressurizadas foi realizada conforme descrito por Garcia (2012). A unidade experimental esta representada na Figura 1, é constituída basicamente de um cilindro de dióxido de carbono, dois banhos termostáticos, duas bombas tipo seringa, um vaso extrator encamisado de aço inox, com capacidade de $507,5 \mathrm{~mL}$, um transdutor absoluto de pressão equipado com um programador portátil e um vaso coletor. Aproximadamente 17 gramas de material seco foram introduzidas no extrator. Em seguida, o solvente foi bombeado e mantido em contato com a matriz por pelo menos 30 minutos para permitir a estabilização do sistema. O óleo foi coletado a partir da abertura da válvula macrométrica e micrométrica. Depois disso, a massa de óleo extraída foi pesada e o tubo foi reconectado ao equipamento. As extrações foram realizadas nas condições descritas na Tabela 1. O fluxo de solvente fixo para o $\mathrm{CO}_{2}$ foi de $3 \mathrm{~mL} \mathrm{~min}^{-1}$ e a coleta da amostra foi de 10 em 10 minutos e após 20 minutos de extração de 20 em 20 minutos. O rendimento de extração foi calculado pela razão entre a massa de óleo extraído e da matéria prima utilizada.

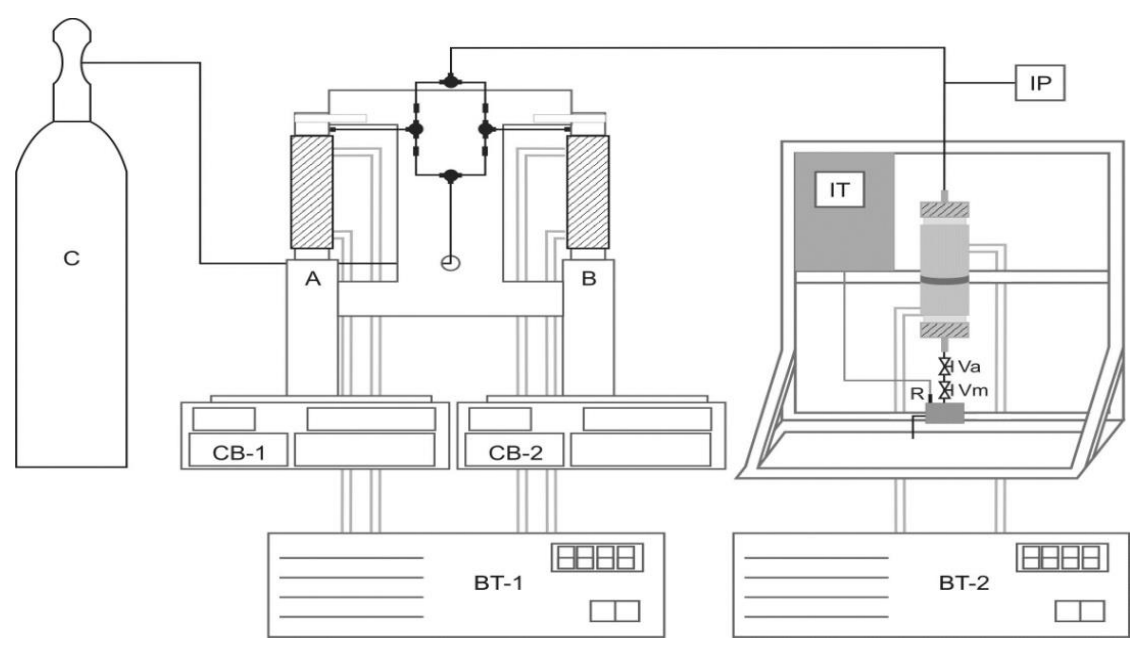

Figura 1 - Aparato experimental utilizado nas extrações por fluido supercrítico. (C) Cilindro de gás; (A) e (B) Bomba tipo seringa; (CB-1) e (CB-2) Controlador das bombas; (BT-1) Banho termostatizado das bombas; (BT-2) Banho termostatizado do extrator; (IT) Indicador de temperatura; (IP) Indicador de pressão; (Va) Válvula agulha; (Vm) Válvula micrométrica; (R1) Reservatório de coleta do óleo (Garcia, 2012).

Tabela 1 - Níveis de temperatura e pressões utilizados nos ensaios de extração do óleo da amêndoa da macaúba

\begin{tabular}{|c|c|c|}
\hline Nível & Temperatura $\left(^{\mathbf{0}} \mathbf{C}\right)$ & Pressão (bar) \\
\hline-1 & 60 & 180 \\
\hline 0 & 70 & 200 \\
\hline+1 & 80 & 220 \\
\hline
\end{tabular}

\subsection{Modelagem Matemática da Cinética de Extração com $\mathrm{CO}_{2}$}

As curvas cinéticas de extração de óleo da amêndoa da macaúba foram ajustadas 
usando o modelo proposto por Sovová (1994). Este modelo considera que a taxa de extração é dividida em três etapas. No primeiro estágio, a taxa de extração é limitada pela transferência de massa do óleo na fase fluida (etapa de taxa constante de extração - CER). No último estágio, a taxa de extração é limitada pela difusão do óleo no sólido. No estágio intermediário, estão presentes ambos os mecanismos de transferência de massa (etapa de taxa decrescente de extração - FER). A solução analítica do modelo Sovová é formada pelas Equações (1)-(3).

Para $t<t_{C E R}$ :

$$
m(t)=\dot{m}_{F} Y_{S} t[1-\exp (-Z)]
$$

Para $t_{C E R} \leq t \leq t_{F E R}$ :

$$
m(t)=\dot{m}_{F} Y_{S}\left[t-t_{C E R} \exp \left(\frac{Z Y_{S}}{W X_{0}} \ln \left\{\frac{1}{1-r}\left(\exp \left(\frac{W \dot{m}_{F}}{m_{s}}\right)\left(t_{C E R}-t\right)-r\right)\right\}-Z\right)\right]
$$

Para $t>t_{\text {FER }}$ :

$$
m(t)=m_{s}\left[X_{0}-\frac{Y_{s}}{W} \ln \left\{1+\left(\exp \left(\frac{W X_{0}}{Y_{S}}\right)-1\right) \exp \left(\frac{W \dot{m}_{F}}{m_{s}}\right)\left(t_{C E R}-t\right) r\right\}\right]
$$

Em que:

$$
\begin{gathered}
Z=\frac{k_{F} a m_{s} \rho_{F}}{\dot{m}_{F} \rho_{S}} \\
W=\frac{m_{s} k_{S} a}{\dot{m}_{F}(1-\varepsilon)} \\
t_{C E R}=\frac{(1-r) m_{s} X_{0}}{Y_{S} Z \dot{m}_{F}} \\
t_{F E R}=t_{C E R}+\frac{m_{s}}{W \dot{m}_{F}} \ln \left[r+(1-r) \exp \left(\frac{W X_{0}}{Y_{S}}\right)\right]
\end{gathered}
$$

Onde: $\dot{m}_{F}$ é a vazão mássica de solvente $\left(\mathrm{M} \mathrm{T}^{-1}\right), Y_{S}$ é a solubilidade do óleo no solvente ( $\left.\mathrm{g}_{\text {óleo }} / \mathrm{g}_{\text {solvente }}\right), t$ é o tempo de extração $(\mathrm{T}), X_{0}$ é a concentração inicial do óleo na matriz sólida $\left(\mathrm{M} \mathrm{M}^{-1}\right), m_{S}$ é a massa de sólido isenta de óleo $(\mathrm{M}), r$ é a fração de óleo facilmente acessível $\left(X_{P} / X_{0}\right), t_{C E R}$ é o tempo que se leva para o término do primeiro período de extração $(\mathrm{T}), t_{F E R}$ se refere ao tempo final do segundo período de extração (T), $k_{F} a$ é o coeficiente de transferência de massa no filme externo $\left(\mathrm{T}^{-1}\right), \varepsilon$ é a porosidade do leito, $k_{S} a$ é o coeficiente de transferência de massa no sólido $\left(\mathrm{T}^{-1}\right), \rho_{F}$ é a densidade do 
fluido $\left(\mathrm{M} \mathrm{L}^{-3}\right), \rho_{S}$ é a densidade do sólido $\left(\mathrm{M} \mathrm{L}^{-3}\right) Z$ e $W$ são os parâmetros adimensionais do modelo.

O parâmetro $r$ é constante devido o processo de preparação (moagem e peneiramento) ser o mesmo para todo o material e este foi ajustado aplicando o método "golden search", com a seguinte função objetivo:

$$
F=\sum_{i=1}^{n-\exp } \sum_{j=1}^{N}\left(m_{o i l_{i, j}}^{\text {Calc }}-m_{o i l_{i, j}}^{E x p}\right)
$$

Os parâmetros $Z$ e $W$ foram determinados com o emprego do método de otimização multivariável Simplex Down-Hill (Nelder and Mead, 1965) a partir da minimização da seguinte função objetivo:

$$
F=\sum_{j=1}^{N}\left(m_{o i l_{j}}^{\text {Calc }}-m_{o i l_{j}}^{E x p}\right)
$$

Onde: $m_{o i l, j}^{\text {Calc }}$ é a massa de óleo extraída obtida pelo modelo de Sovová; $m_{o i l, j}^{E x p}$ é a massa de óleo obtida experimentalmente, $n_{-}$exp é o número experimentos e $N$ o número de pontos da curva cinética experimental.

\section{RESULTADOS E DISCUSSÃO}

A Tabela 2 apresenta as condições experimentais, solubilidade do óleo em $\mathrm{CO}_{2} \mathrm{e}$ rendimento nas condições avaliadas, bem como os resultados para extração utilizando $n$ hexano e diclorometano como solventes. Verifica-se que o aumento da pressão ocasiona o aumento da densidade do solvente e da solubilidade do óleo no solvente, resultando em maiores rendimentos de óleo. Garcia et al. (2012) e Nimet et al. (2011) relataram o efeito positivo da extração, com o aumento da pressão sobre a taxa rendimento com $\mathrm{CO}_{2}$ supercrítico na extração do óleo de sementes de Mucuna e girassol, respectivamente. $\mathrm{O}$ aumento da temperatura exerceu efeito negativo sobre o rendimento em óleo, devido o decréscimo na densidade do solvente.

O melhor rendimento obtido por extração supercrítica com $\mathrm{CO}_{2}$ do óleo da amêndoa da macaúba foi de $41,55 \%$ na condição de $40{ }^{\circ} \mathrm{C}$ e 220 bar, sendo que esta condição apresentou rendimento $>93 \%$ do obtido pelo método convencional com $n$-hexano e diclorometano. Na extração realizada pelo método de Soxhlet com $n$-hexano e diclorometano o rendimento foi de $43,49 \%$ e 44,49\% respectivamente. Hiane et al. (2005) e Coimbra e Jorge (2011a) reportam teores de óleo da amêndoa da macaúba de 52,9\% e $46,06 \%$, respectivamente. Em ambos os trabalhos foi utilizado método de Soxhlet e como solvente o éter de petróleo.

Os parâmetros utilizados para a modelagem matemática da cinética de extração foram: concentração inicial de óleo de $0,712 \mathrm{~g}_{\text {óleo }} \mathrm{g}_{\text {sólido }}{ }^{-1}$ (dada pela razão entre a massa de óleo nas sementes e a massa de sólido inerte), densidade do sólido de $1,003 \mathrm{~g} \mathrm{~cm}^{-3}$, densidade do leito de $0,092 \mathrm{~g} \mathrm{~cm}^{-3}$, porosidade de 0,908 , massa de material inerte de $9,93 \mathrm{~g}$, vazão mássica de solvente de $2,9 \times 10^{-3} \mathrm{~kg} \mathrm{~min}^{-1}$, densidade do solvente e solubilidade do óleo no solvente de 
acordo com a Tabela 2. A solubilidade $\left(Y_{S}\right)$ do óleo no solvente foi determinada pelo método dinâmico através do coeficiente angular da parte linear da curva de extração.

Tabela 2 - Condições experimentais das extrações do óleo com $\mathrm{CO}_{2}$ supercrítico e rendimentos

\begin{tabular}{|c|c|c|c|c|c|}
\hline Experimento & $\begin{array}{c}\mathrm{T} \\
\left({ }^{\circ} \mathrm{C}\right)\end{array}$ & $\begin{array}{c}\mathrm{P} \\
(\mathrm{bar})\end{array}$ & $\begin{array}{c}\rho_{F} \\
\left(\mathrm{~g} \mathrm{~cm}^{-3}\right)\end{array}$ & $\begin{array}{c}Y_{S} \\
\left(\mathrm{~g}_{\text {óleo }} \mathrm{g}_{\text {solvente }}{ }^{-1}\right)\end{array}$ & $\begin{array}{c}\text { Rendimento } \\
(\%)\end{array}$ \\
\hline 1 & 40 & 180 & 0,82029 & 0,0156 & 36,87 \\
\hline 2 & 40 & 220 & 0,85811 & 0,0267 & 41,55 \\
\hline 3 & 80 & 180 & 0,53974 & 0,0039 & 9,31 \\
\hline 4 & 80 & 220 & 0,63705 & 0,0108 & 27,23 \\
\hline 5 & 60 & 200 & 0,72411 & 0,0125 & $30,8^{\mathrm{a}} \pm 0,23$ \\
\hline \multirow{2}{*}{ Soxhlet } & \multicolumn{4}{|c|}{$n$-hexano } & $43,64^{\mathrm{a}} \pm 0,48$ \\
\cline { 2 - 6 } & \multicolumn{4}{|c|}{ Diclorometano } & $44,49^{\mathrm{a}} \pm 0,78$ \\
\hline
\end{tabular}

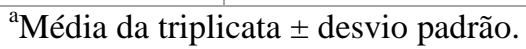

Os parâmetros ajustados do modelo de Sovová são apresentados na Tabela 3. O coeficiente de transferência de massa no filme externo, $k_{F a}$, foi obtido usando o valor de $Z$ e a Equação 4, enquanto que o coeficiente de transferência de massa no sólido, $k_{S a}$, empregando $W$ e a Equação 5. O parâmetro $r$ (fração de óleo presente na superfície das células) é constante, ou seja, considera-se a eficiência da moagem como sendo a mesma para todas as sementes devido à utilização da mesma granulometria nos experimentos.

Tabela 3 - Parâmetros ajustados do modelo de Sovová para as extrações do óleo com $\mathrm{CO}_{2}$ supercrítico

\begin{tabular}{|c|c|c|c|c|c|c|c|c|}
\hline $\begin{array}{c}\mathrm{T} \\
\left({ }^{\circ} \mathrm{C}\right)\end{array}$ & $\begin{array}{c}\mathrm{P} \\
(\mathrm{bar})\end{array}$ & $Z$ & $W$ & $r$ & $\begin{array}{c}t_{C E R} \\
(\mathrm{~min})\end{array}$ & $\begin{array}{c}t_{F E R} \\
(\mathrm{~min})\end{array}$ & $\begin{array}{c}k_{F a} \\
\left(\mathrm{~min}^{-1}\right)\end{array}$ & $\begin{array}{c}k_{S a} \\
\left(\mathrm{~min}^{-1}\right)\end{array}$ \\
\hline 40 & 180 & 45.9952 & 0.0610 & 0.8537 & 0.4878 & 64.6733 & 1.5234 & 0.0017 \\
\hline 40 & 220 & 45.9016 & 0.0811 & 0.8537 & 0.2876 & 31.7577 & 1.4460 & 0.0022 \\
\hline 80 & 180 & 1.9745 & 0.0040 & 0.8537 & 45.9166 & 170.0620 & 0.0984 & 0.0001 \\
\hline 80 & 220 & 3.4317 & 0.0310 & 0.8537 & 9.6562 & 85.0842 & 0.1431 & 0.0008 \\
\hline 60 & 200 & 20.2136 & 0.0437 & 0.8537 & 1.3991 & 75.6536 & 0.7509 & 0.0012 \\
\hline
\end{tabular}

O maior valor de $k_{S a}$ foi obtido na condição de menor temperatura e maior pressão $\left(40{ }^{\circ} \mathrm{C}\right.$ e 220 bar), evidenciando o maior rendimento obtido neste experimento bem como a 
cinética mais rápida. Isso se deve ao fato deste parâmetro representar a facilidade da extração do óleo de difícil acesso (etapa limitante do processo). Ainda, pode-se relacionar este parâmetro à densidade do $\mathrm{CO}_{2}$, sendo tanto maior quanto maior o valor da densidade.

As curvas cinéticas experimentais de extração com o ajuste do modelo de Sovová são apresentadas na Figura 2, onde constatou-se um coeficiente de correlação $\left(R^{2}\right)>0,99$ para todas as condições experimentais.

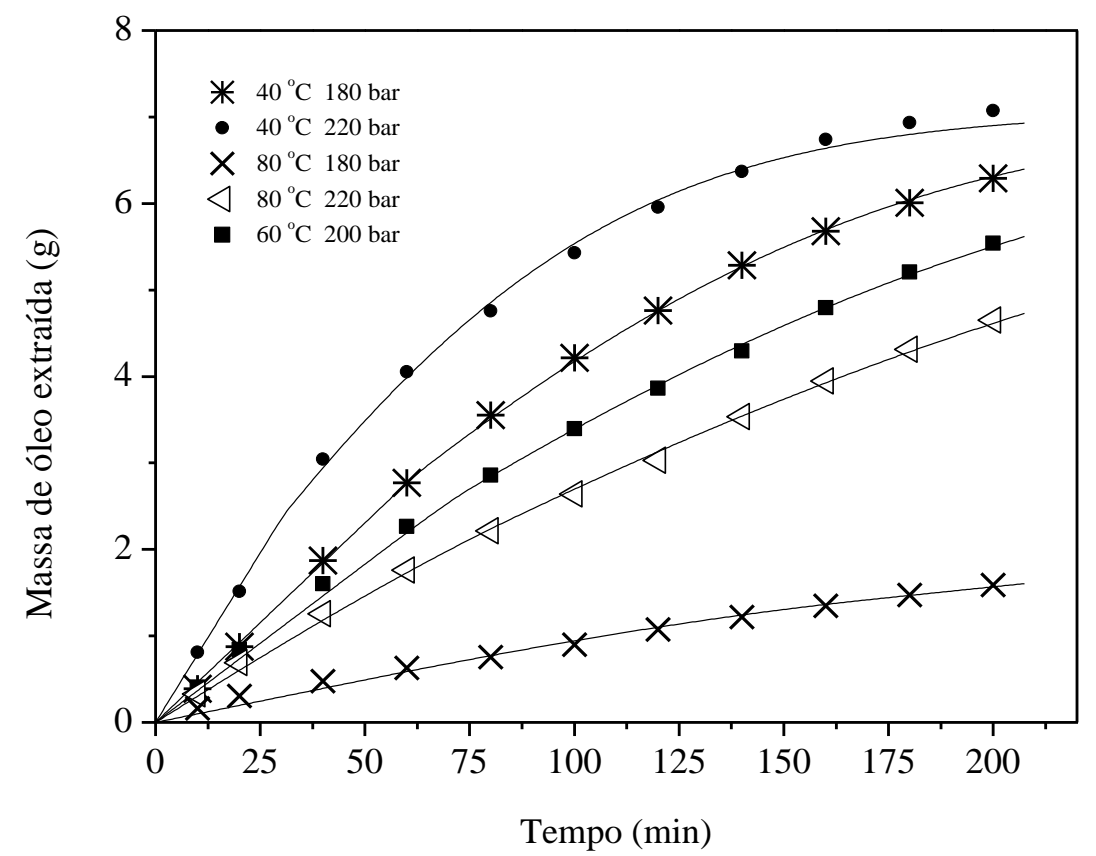

Figura 2 - Cinéticas experimentais de extração com ajuste do modelo de Sovová.

\section{CONCLUSÕES}

Os resultados demonstraram o elevado rendimento na extração supercrítica na condição de $40{ }^{\circ} \mathrm{C}$ e 220 bar, condição que apresentou a maior densidade do $\mathrm{CO}_{2}$. Podendo observar-se pelas cinéticas de extração que quanto maior a densidade maior foi o rendimento do óleo extraído. O maior rendimento na extração supercrítica $(41,55 \%)$ ficou próximo dos rendimentos da extração por Soxhlet utilizando com solvente o $n$-hexano $(43,49 \%)$ e diclorometano $(44,49 \%)$. O modelo Sovová descreveu satisfatoriamente a cinética do processo de extração do óleo da amêndoa da macaúba.

\section{REFERÊNCIAS}

ARIAS, M.; PENICHET, I.; YSAMBERTT, F.; BAUZA, R.; ZOUGAGH, M. RIOS; A. Fast supercritical fluid extraction of low and high density polyethylene additives: Comparison with conventional reflux and automatic soxhlet extraction. $J$. Supercrit. Fluids, v. 50, p. 22-28, 2009.

CICONINI, G.; FAVARO, S.P.; ROSCOE, R.; MIRANDA C.H.B.; TAPETI, C.F.; MIYAHIRA, M.A.M.; BEARARI, L.; GALVANI, F.; BORSATO, A.V., COLNAGO, L.A.; NAKA, M.H. Biometry and oil contents of Acrocomia aculeata fruits from the Cerrados and Pantanal biomes in Mato Grosso do Sul, 
Brazil. Ind. Crops and Prod., v. 45, p. 208-214, 2013.

CORSO, M.P.; SILVA, E.A.; KLEN, M.R.F.; CARDOZO-FILHO, L.; SANTOS, J.N.; FREITAS, L.S.; DARIVA, C. Extraction of sesame seed (Sesamun indicum L.) oil using compressed propane and supercritical carbon dioxide. J. Supercrit. Fluids, v. 52 , p. $56-61,2010$.

GARCIA,V.A.S.; CABRAL, V.F.; ZANOELO, E.F.; SILVA, C.; CARDOZO-FILHO, L. Extraction of Mucuna seed oil using supercritical carbon dioxide to increase the concentration of 1-Dopa in the defatted meal. J. Supercrit. Fluids, v. 69, p. 75$81,2012$.

GARCIA, V.A.S. Extração de óleo de Mucuna utilizando dióxido de carbono supercrítico visando concentrar farelo desengordurado em L-Dopa. 106f, 2012. (Dissertação de mestrado) - Centro de ciências agrárias, Universidade estadual de Maringá, Maringá, 2012.

HUISDEN, C.M.; ADESOGAN, A.T.; GASKIN, J.M.; COURTNEY, C.H.; RAJI, A. M.; KANG, T. Effect of feeding Mucuna pruriens on helminth parasite infestation in lambs. J. Ethnopharm., v. 127, p. 669-673, 2010.

INSTITUTO ADOLFO LUTZ. Métodos Físico-Químicos para Análise de Alimentos $4^{\text {a }}$ Edição, $1^{\text {a }}$ edição digital, 2004.

LORENZI, G.M.A.C.; NEGRELLE, R.R.B. Acrocomia aculeata (Jacq.) Lodd. ex Mart: aspectos ecológicos, usos e potencialidades. Visão Acad., v.7, 2006.

MERCK. Ficha de informações de segurança de produtos químicos: $n$-hexano. São Paulo: Merck S. A. 2006.

NIMET, G.; SILVA, E.A.; PALÚ, F.; DARIVA, C.; FREITAS, L.S.; NETO, A.M.; CARDOZO-FILHO, L. Extraction of sunflower (Heliantus annuus L.) oil with supercritical $\mathrm{CO} 2$ and subcritical propane: Experimental and modeling. Chem. Eng. J., v. 168, p. $262-268,2011$.

PEDERSSETTI, M.M.; PALÚ, F.; SILVA, E.A.; ROHLING, J.H.; CARDOZO-FILHO, L.; DARIVA, C. Extraction of Canola Seed (Brassica napus) Oil using Compressed Propane and Supercritical Carbon Dioxide. J. Food Eng., v. 102, p. 189-196, 2010.

PRADO, A. G. S. Química Verde, Os Desafios da Química do Novo Milênio. Quím. Nova, v.26, p.738-744, 2003.

REVERCHON, E.; DE MARCO, I. Supercritical fluid extraction and fractionation of natural matter. J. Supercrit. Fluids, v. 38, p. 146-166, 2006.

SOVOVÁ, H. Rate of Vegetable Oil Extraction with Supercritical CO2 - I and II Modelling of Extraction Curves. Chem. Eng. Scienc., v. 49, n. 3, p. 409,1994.

SUN, H.; GE, X.; LV, Y.; WANG, A. Application of accelerated solvent extraction in the analysis of organic contaminants, bioactive and nutritional compounds in food and feed. J. Chromat., v.12, p. 1-23, 2012. 